\title{
A fuzzy goal programming approach to solving decentralized bi-level multi-objective linear fractional programming problems
}

\author{
Tunjo Perić ${ }^{1, *}$, Zoran Babić ${ }^{2}$, Maid Omerović ${ }^{3}$ \\ ${ }^{1}$ Faculty of Economics and Business, University of Zagreb \\ Trg J. F. Kennedya 6, 10000 Zagreb, Croatia \\ E-mail: 〈tperic@efzg.hr〉 \\ ${ }^{2}$ Faculty of Economics, Business and Tourism, University of Split \\ Cvite Fiskovića 5, 21000 Split, Croatia \\ E-mail:〈babic@efst.hr〉 \\ ${ }^{3}$ Faculty of Education, University of Travnik \\ Aleja konzula 5, 72270 Travnik, Bosnia and Herzegovina \\ E-mail:〈maid.omerovic@gmail.com〉
}

\begin{abstract}
This paper presents a new approach for solving decentralized bi-level multi-objective linear fractional programming problems. The main goal was to find a simple algorithm with high confidence of decision-makers in the results. First, all the linear fractional programming models on the given set of constraints were solved separately. Next, all the linear fractional objective functions were linearized, membership functions of objective functions and decision variables controlled by decision-makers at the highest level calculated, and a fuzzy multi-objective linear programming model formed and solved as linear goal programming problem by using simplex algorithm. The efficiency of the proposed algorithm was investigated using an economic example, and the obtained results compared with those obtained using an existing method.
\end{abstract}

Keywords: bi-level programming, linear fractional programming, multiple objective, production plan

Received: October 31, 2018; accepted: February 4, 2019; available online: July 4, 2019

DOI: $10.17535 /$ crorr.2019.0007

\section{Introduction}

Decentralized complex hierarchical systems have two or more decision-making levels. Decisions made at the highest level determine the frameworks of decisions made at lower levels. Decisionmakers at both levels try to maximize utility functions on given sets of constraints. They also try to control variables. Decisions made by a decision-maker (DM) at the higher level influences those made by DMs at the lower level. In addition, decisions made by DMs at the lower level affect the degree to which decisions are achieved at the higher level. Equilibrium is achieved when all DMs simultaneously realize their utility function maximum on the given sets of constraints.

This method of decision-making is complex. The DM at the higher level must have complete information on the goals, constraints, and parameters of the DMs at the lower level in order to make a decision to achieve his own maximum utility function, while also achieving the maximum utility functions of the DMs at lower levels. When a decentralized model contains only two DMs at two levels, the decision-making problem can be presented as a sequential model

${ }^{*}$ Corresponding author. 
with complete information, known as Stackelberg's model [23]. Bi-level programming includes inverse induction in the procedure of problem-solving. Solving bi-level programming problems using Stackelberg's model causes several mathematical problems related to non-convexity and disconnectedness, even for simpler bi-level programming problems, leading to obtaining dominated solutions in many situations [20]. Bi-level programming is an NP-hard problem [6]. Many methods have been developed to solve bi-level programming problems. An overview is presented in [20]. Most of the methods developed are unsuitable for solving real problems. Solving bi-level programming problems becomes more complicated when DMs have more objective functions, especially when objective functions and/or constraints are non-linear.

Here we propose a methodology for solving decentralized, bi-level, multi-objective, linear fractional programming (DBL-MOLFP) problems. It includes two methods: i) a method for the linearization of linear fractional objective functions, and ii) fuzzy goal programming (FGP). The proposed methodology should improve the decision-making process in decentralized business systems with conflicting goals, which would lead to the better functioning of the entire complex system. The efficiency of the proposed methodology is presented using an example of production plan, inventory and promotion cost determination for a company.

The rest of the paper is organized as follows. Section 2 gives literature review. In Section 3 the methodology for solving a DBL-MOLFP problem is presented. Section 4 gives an application example, and the conclusion, in the last section (Section 5), gives the main research results.

\section{Literature review}

In order to avoid problems solving bi-level and multi-level programming problems caused by methods based on Stackelberg's model, many authors see them as multi-objective programming problems in which decision-makers try to maximize objective functions while simultaneously controlling variables. A number of papers refer to decentralized bi-level multi-objective linear programming (DBL-MOLP) problems [2, 3, 4, 15, 17, 18, 19, 21, 22].

Sakawa and Nishizaki [16] propose an interactive fuzzy programming methodology to solve two-level linear fractional programming problems with essentially cooperative decision-makers. A satisfactory solution is obtained by changing the satisfactory level of the decision-maker at the higher level, providing a satisfactory balance between levels overall. Ahlatcioglu and Tiryaki [1] present two interactive fuzzy programming methodologies to solve decentralized two-level linear fractional programming problems with one DM at the upper level and multiple DMs at the lower level. In the first methodology, the decision-maker at the highest level $\left(D M_{0}\right)$ determines the minimal satisfactory level of his objective function to form membership function, and decreases it to improve the objective functions of DMs at the lower level. The second methodology extends the first by including the membership functions for upper-level decision variables.

Mishra [9] uses a weighting method to solve the DBL-LFP problem. Here, the weights are calculated using the Analytic Hierarchy Process (AHP) method and the objective functions of both levels are included in one objective function. Lachhwani and Poonia [8] use a fuzzy goal programming method to solve multilevel fractional programming problems. Emam [5] proposes an interactive approach to solving bi-level multi-objective fractional programming problems. It begins by finding the convex hull of the original constraints set using a cutting plane algorithm, and the Charnes-Cooper transformation to linearize the fractional objective functions. In the second phase, the problem is transformed into a separate multi-objective linear programming problem and solved by the $\varepsilon$-constraint method.

Lachhwani [7] presents a new method based on fuzzy goal programming to solve multi-level, multi-objective, linear fractional programming problems. The numerator and denominator functions of each objective function are transformed into fuzzy goals and different linear membership functions are determined for the numerator and denominator of each objective function. Toksari and Bilim, [24] present an interactive fuzzy goal programming method to solve decen- 
A fuzzy goal programming approach to solving decentralized bi-level multi-objective...

tralized bi-level multi-objective fractional programming (DBL-MOFP) problems. The membership functions of all objective functions are linearized using the Jacobian matrix and then fuzzy goal programming is solved. Osman, Raslan and Emam [11] use a fuzzy goal programming model to solve ML-MOFP problems with rough intervals in the objective functions.

\section{Methodology for solving DBL-MOLFP problems}

\subsection{DBL-MOLFP model}

Let suppose that decision model consists of two levels, the upper and lower level, where one decision-maker is at the upper level $\left(D M_{0}\right)$ and $p$ decision-makers at the lower level $\left(D M_{i}, i=\right.$ $1,2, \ldots, p)$. The model contains variable vectors $\mathbf{x}=\left(\mathbf{x}_{\mathbf{0}}, \mathbf{x}_{\mathbf{1}}, \ldots, \mathbf{x}_{\mathbf{p}}\right) \in \mathbb{R}^{n}, \mathbf{x}_{\mathbf{0}} \in \mathbb{R}^{n_{0}}, \mathbf{x}_{\mathbf{k}} \in \mathbb{R}^{n_{k}}$, where $n=n_{0}+n_{1}+\ldots+n_{p}, \mathbf{x}_{\mathbf{0}}=\left(x_{01}, x_{02}, \ldots, x_{0 n_{0}}\right)$ and $\mathbf{x}_{\mathbf{k}}=\left(x_{k 1}, x_{k 2}, \ldots, x_{k n_{k}}\right)$ for $k=1,2, \ldots, p$. Decision maker $D M_{0}$ controls variables $\mathbf{x}_{\mathbf{0}}$, decision maker $D M_{1}$ controls variables $\mathbf{x}_{\mathbf{1}}$, etc. The DBL-MOLFP model can be presented as follows:

$$
\begin{gathered}
z_{o}=\max _{\mathbf{x} \in \mathbf{S}} \mathbf{z}_{\mathbf{0}}(\mathbf{x})=\frac{c_{0}^{0} \mathbf{x}_{\mathbf{0}}+c_{0}^{1} \mathbf{x}_{\mathbf{1}}+\cdots+c_{0}^{p} \mathbf{x}_{\mathbf{p}}+\alpha_{0}}{d_{0}^{0} \mathbf{x}_{\mathbf{0}}+d_{0}^{1} \mathbf{x}_{\mathbf{1}}+\cdots+d_{0}^{p} \mathbf{x}_{\mathbf{p}}+\beta_{0}}, \\
z_{1}=\max _{\mathbf{x} \in \mathbf{S}} \mathbf{z}_{\mathbf{1}}(\mathbf{x})=\frac{c_{0}^{0} \mathbf{x}_{\mathbf{0}}+c_{0}^{1} \mathbf{x}_{\mathbf{1}}+\cdots+c_{0}^{p} \mathbf{x}_{\mathbf{p}}+\alpha_{1}}{d_{0}^{0} \mathbf{x}_{\mathbf{0}}+d_{0}^{1} \mathbf{x}_{\mathbf{1}}+\cdots+d_{0}^{p} \mathbf{x}_{\mathbf{p}}+\beta_{1}}, \\
z_{2}=\max _{\mathbf{x} \in \mathbf{S}} \mathbf{z}_{\mathbf{2}}(\mathbf{x})=\frac{c_{0}^{0} \mathbf{x}_{\mathbf{0}}+c_{0}^{1} \mathbf{x}_{\mathbf{1}}+\cdots+c_{0}^{p} \mathbf{x}_{\mathbf{p}}+\alpha_{2}}{d_{0}^{0} \mathbf{x}_{\mathbf{0}}+d_{0}^{1} \mathbf{x}_{\mathbf{1}}+\cdots+d_{0}^{p} \mathbf{x}_{\mathbf{p}}+\beta_{2}}, \\
\vdots \\
z_{p}=\max _{\mathbf{x} \in \mathbf{S}} \mathbf{p}_{\mathbf{2}}(\mathbf{x})=\frac{c_{0}^{0} \mathbf{x}_{\mathbf{0}}+c_{0}^{1} \mathbf{x}_{\mathbf{1}}+\cdots+c_{0}^{p} \mathbf{x}_{\mathbf{p}}+\alpha_{p}}{d_{0}^{0} \mathbf{x}_{\mathbf{0}}+d_{0}^{1} \mathbf{x}_{\mathbf{1}}+\cdots+d_{0}^{p} \mathbf{x}_{\mathbf{p}}+\beta_{p}},
\end{gathered}
$$

where $\mathbf{S}$ is non empty set:

$$
\begin{gathered}
\mathbf{S}=\left\{\mathbf{x} \in \mathbb{R}^{n}: \mathbf{A}_{\mathbf{0}} \mathbf{x}_{\mathbf{0}}+\mathbf{A}_{\mathbf{1}} \mathbf{x}_{\mathbf{1}}+\cdots+\mathbf{A}_{\mathbf{p}} \mathbf{x}_{\mathbf{p}}(\leq,=, \geq) \mathbf{b}\right. \\
\left.\mathbf{x}=\left[\mathbf{x}_{\mathbf{0}}, \mathbf{x}_{\mathbf{1}}, \ldots, \mathbf{x}_{\mathbf{p}}\right]^{T} \geq 0, \quad \mathbf{b} \in \mathbb{R}^{m}\right\}=\varnothing
\end{gathered}
$$

and $c_{k}^{0}, c_{k}^{1}, \ldots, c_{k}^{p}, d_{k}^{0}, d_{k}^{1}, \ldots, d_{k}^{p}$ are vectors of coefficients of the numerator and denominator of the $k$-th objective function, $\mathbf{c}_{k}^{i}=\left(c_{k 1}^{i}, c_{k 2}^{i}, \ldots, c_{k n_{k}}^{i}\right), \mathbf{d}_{k}^{i}=\left(d_{k 1}^{i}, d_{k 2}^{i}, \ldots, d_{k n_{k}}^{i}\right) i, k=0,1, \ldots, p$ are vector coefficients (with components) of objective functions, $\alpha_{k}$ and $\beta_{k}$ are constants in objective functions, $\mathbf{A}_{0}, \mathbf{A}_{i}, i=1,2, \ldots, p$ are the matrices of the constraint coefficients, and $\mathbf{o}$ is a null vector. The coefficients $\mathbf{c}$ and $\mathbf{d}$ may be sales prices per unit, gross profit per unit, inventory cost per unit, etc. $\alpha$ and $\beta$ are constants that may be fixed costs, fixed income, etc. The variables $\mathbf{x}$ in the model can be the number of product units, stock quantity of products, investment in promotion of products, etc.

\subsection{Linear fractional objective function linearization and objective function weights determination}

To solve bi-level, multi-objective, linear fractional programming problems using the linear goal programming method, linear fractional objective functions must be linearized. This can be done using different methods $[12,13,14]$. Here we present the linearization method in [12].

The following linearization is proposed: 


$$
\begin{aligned}
& \frac{\mathbf{c}_{k} \mathbf{x}+\alpha_{k}}{\mathbf{d}_{k} \mathbf{x}+\beta_{k}} \leq(\geq) \overline{\mathbf{z}}_{k}, \quad \mathbf{d}_{k} \mathbf{x}+\beta_{k}>0 \quad \forall \mathbf{x}, \quad k=0,1, \ldots, p \\
& \mathbf{c}_{k} \mathbf{x}+\alpha_{k} \leq(\geq) \overline{\mathbf{z}}_{k}\left(\mathbf{d}_{k} \mathbf{x}+\beta_{k}\right) \\
& \left(\mathbf{c}_{k}-\overline{\mathbf{z}}_{k} \mathbf{d}_{k}\right) \mathbf{x} \leq(\geq) \overline{\mathbf{z}}_{k} \beta_{k}-\alpha_{k} \\
& \mathbf{C}_{k} \mathbf{x}-\overline{\mathbf{Z}}_{k} \leq(\geq) 0 \\
& \mathbf{z}_{k, \text { lin }}=\mathbf{C}_{k} \mathbf{x}-\overline{\mathbf{Z}}_{k}, \quad k=1,2, \ldots, K .
\end{aligned}
$$

where $\overline{\mathbf{z}}_{k}$ is the aspired acceptable value of the kth objective function determined by the decision-maker, or its optimal value, $\mathbf{C}_{k}=\mathbf{c}_{k}-\overline{\mathbf{z}}_{k} \mathbf{d}_{k}, \overline{\mathbf{Z}}_{k}=\overline{\mathbf{z}}_{k} \beta_{k}-\alpha_{k}$, and $\mathbf{z}_{k, \text { lin }}$ is the linearized objective function $\mathbf{z}_{k}, k=0,1, \ldots, K$.

The objective function weights should enable an objective function with a higher weight to be met more than an objective function with a lower objective function weight. To determine the weights of the objective functions, we propose using the AHP method, so that decisionmaker $D M_{0}$ compares the importance of the function $z_{0}$ with functions $z_{1}, z_{2}, \ldots, z_{p}$, decisionmaker $D M_{1}$ compares objective function $z_{1}$ with functions $z_{2}, z_{3}, \ldots, z_{p}$, decision-maker $D M_{2}$ compares objective function $z_{2}$ with functions $z_{3}, z_{4}, \ldots, z_{p}$, etc. The objective function weights are calculated in three steps:

Step 1. The DMs evaluate objective functions by comparing their objective function with the remaining objective functions using Satty's scale (from 1 to 9 ). Their evaluations are marked with $e_{i j}$, where $e_{i j}=1$ for $i=j$ and $e_{i j}=e_{j i}$ for $i \neq j$.

Step 2. Calculate $d_{i j}=\frac{e_{i j}}{\sum_{i=0}^{p} e_{i j}} \forall j=0,1, \ldots, p$

Step 3. Calculate the objective functions weights $w_{0}=\frac{\sum_{j=0}^{p} d_{0 j}}{p+1}, \ldots, w_{p}=\frac{\sum_{j=0}^{p} d_{p j}}{p+1}$.

\subsection{Membership of objective functions and decision variables}

This paper proposes a fuzzy goal programming methodology to solve DBL-MOLFP problems in which DMs must determine the acceptable value of the objective functions, and the DM at the higher level must determine the allowed negative $\left(t_{0 j}^{L}\right)$ and positive $\left(t_{0 j}^{R}\right)$ deviation of the decision variables they control. On this basis, the membership functions of the linearized objective functions and the decision variables controlled by the decision maker $D M_{0}$ are calculated:

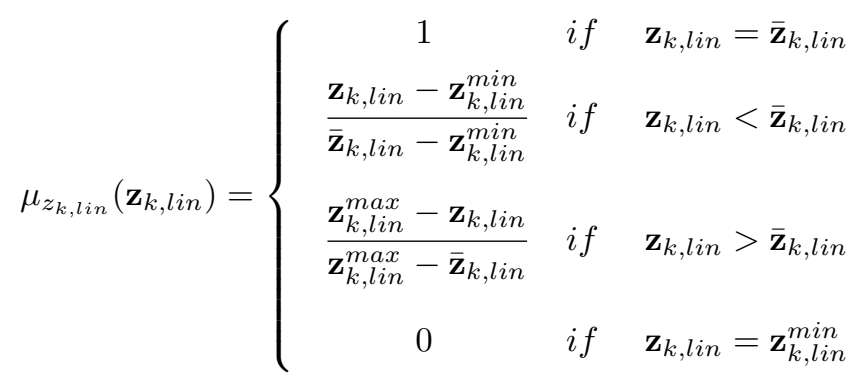

$$
\begin{aligned}
& \mu_{x_{0 j}}\left(x_{0 j}\right)=\left\{\begin{array}{cl}
\mu_{0 j}^{L}\left(x_{0 j}\right)=\frac{x_{0 j}-\left(x_{0 j}^{*}-t_{0 j}^{L}\right)}{t_{0 j}^{L}} & \text { if } \quad\left(x_{0 j}^{*}-t_{0 j}^{L}\right) \leq x_{o j} \leq x_{0 j}^{*} \\
\mu_{0 j}^{R}\left(x_{0 j}\right)=\frac{\left(x_{0 j}^{*}+t_{0 j}^{R}\right)-x_{0 j}}{t_{0 j}^{R}} & \text { if } \quad\left(x_{0 j}^{*} \leq x_{0 j} \leq\left(x_{0 j}^{*}+t_{0 j}^{R}\right)\right. \\
0 & \text { otherwise }
\end{array}\right.
\end{aligned}
$$




\subsection{Decentralized bi-level fuzzy goal programming (DBL-FGP) model}

The DBL-FGP model looks like this:

$$
\min _{\mathbf{x} \in \mathbf{S}_{1}}\left(\sum_{k=0}^{p} w_{k}\left(d_{k}^{-}+d_{k}^{+}\right)+\sum_{j=1}^{n_{0}} w_{x_{0 j}}\left(\mu_{x_{0 j}}^{L}+\mu_{x_{0 j}}^{R}\right)\right)
$$

where

$$
\begin{aligned}
& \mathbf{S}_{\mathbf{1}}=\left\{(\mathbf{x}, \mathbf{d}): \mu_{z_{k, l i n}}\left(\mathbf{z}_{k, l i n}\right)+d_{k}^{-}-d_{k}^{+}=1, \quad k=0,1, \ldots, p\right. \\
& \mathbf{A}_{\mathbf{0}} \mathbf{x}_{\mathbf{0}}+\mathbf{A}_{\mathbf{1}} \mathbf{x}_{\mathbf{1}}+\cdots+\mathbf{A}_{\mathbf{p}} \mathbf{x}_{\mathbf{p}}(\leq,=, \geq) \mathbf{b}, \quad \mathbf{b} \in \mathbb{R}^{m}, \\
&\left.\mathbf{x}=\mathbf{x}_{\mathbf{0}}+\mathbf{x}_{\mathbf{1}}+\cdots+\mathbf{x}_{\mathbf{p}} \geq 0, \quad d_{k}^{-}, d_{k}^{+}, \lambda \geq 0, \quad d_{k}^{-} d_{k}^{+}=0\right\}=\varnothing,
\end{aligned}
$$

Model in eq.(6) can be converted into a linear programming problem as $\underset{\mathbf{x}, \mathbf{d}, \lambda \in \mathbf{S}_{\mathbf{2}}}{\min } \lambda$, and solvable by the simplex method, where

$$
\begin{aligned}
\mathbf{S}_{\mathbf{2}}=\{ & (\mathbf{x}, \mathbf{d}, \lambda): \mu_{z_{k, l i n}}\left(\mathbf{z}_{k, l i n}\right)+d_{k}^{-}-d_{k}^{+}=1, \quad \lambda-d_{k}^{-}-d_{k}^{+} \leq 0, \quad k=0,1, \ldots, p \\
& \lambda+w_{k} d_{k}^{-}+w_{k} d_{k}^{+} \leq 1, \lambda \leq \mu_{x_{0 j}}^{L}\left(x_{0 j}\right), \lambda \leq \mu_{x_{0 j}}^{R}\left(x_{0 j}\right), \quad j=1,0, \ldots, n_{0} \\
& \mathbf{A}_{\mathbf{0}} \mathbf{x}_{\mathbf{0}}+\mathbf{A}_{\mathbf{1}} \mathbf{x}_{\mathbf{1}}+\cdots+\mathbf{A}_{\mathbf{p}} \mathbf{x}_{\mathbf{p}}(\leq,=, \geq) \mathbf{b}, \quad \mathbf{b} \in \mathbb{R}^{m}, \\
& \left.\mathbf{x}=\mathbf{x}_{\mathbf{0}}+\mathbf{x}_{\mathbf{1}}+\cdots+\mathbf{x}_{\mathbf{p}} \geq 0, \quad d_{k}^{-}, d_{k}^{+}, \lambda \geq 0, \quad d_{k}^{-} d_{k}^{+}=0\right\}=\varnothing
\end{aligned}
$$

If the obtained solution is a non empty set, the DMs should accept the obtained solution as preferred, since it is calculated based on information obtained from them. If the obtained solution is an empty set, the DMs should change the aspired acceptable value of the objective functions or/and the allowed negative $\left(t_{0 j^{L}}\right)$ and positive $\left(t_{0 j^{R}}\right)$ deviation of the decision variables they control. To determine the acceptable values of the objective functions that will not cause decision deadlocks in the process of obtaining the preferred solution, DMs should choose the acceptable value of the objective functions between their minimum and maximum values presented in the payoff table. Consequently, decision-makers in a decentralized business system should cooperate in modelling and base their decisions on the obtained results. This should lead to the optimal functioning of the entire business system.

\subsection{Algorithm to solve DBL-MOLFP problem}

To solve the DBL-MOLFP problem, we propose the FGP methodology presented by the following algorithm:

Step 1. Determine the individual optimal value of all objective functions on the set of constraints $\mathbf{S}$ and form the pay-off table.

Step 2. Determine the acceptable value $\overline{\mathbf{z}}_{k}$ of the objective function $\mathbf{z}_{k}$.

Step 3. Linearize all objective functions using the linearization form in eq.(3).

Step 4. Determine acceptable negative and positive deviations $t_{0 j}^{L}$ and $t_{0 j}^{R}$ for $j=1,2, \ldots, n_{0}$ for all decision variables controlled by the decision-maker at the highest level, and form fuzzy membership functions $\mu_{x_{0 j}}^{L}$ and $\mu_{x_{0 j}}^{R}$ using forms in eq.(4) and eq.(5).

Step 5. Using the AHP method, determine the weights of the objective functions.

Step 6. Form the DBL-FGP model in eq.(6).

Step 7. Form and solve the LP model in eq.(8) to obtain a solution to the DBL-MOLFP problem. 
Step 8. If the obtained solution is not an empty set, go to Step 9, otherwise go to Step 10.

Step 9. Stop. A satisfactory solution of the DBL-MOLFP problem is obtained.

Step 10. Modify the acceptable aspiration levels of the objective functions and the positive and negative deviation of the decision variables controlled by decision-maker $D M_{0}$, determine the new membership functions and go back to Step 4.

\section{Practical example}

Consider a company that manufactures five products: P1, P2, P3, P4, P5. Suppose that the costs and required capital are proportional to individual activities. The company requires that all products should be produced in at least 50 units. In addition, the company should stock no less than $12 \%$ of its total production to ensure delivery safety. The management of the enterprise has set the inventory limit for each product to at least 14, 11, 9, 15, and 13, to at most $50 \%$ of total production, and to at most $44,56,52,45$, and 50 for the respective products. It is also stipulated that total inventory costs per product should not exceed 100 m.u. (monetary units). Enterprise management has determined that investment in promotion must not exceed $20 \%$ of the total gross profit of the company. Per product, promotion investments must not exceed $22,18,24,20$, and $19 \%$ respectively of the total gross profit of the company, while promotion investments for the first product may not exceed $800 \mathrm{~m} . \mathrm{u}$. and $400 \mathrm{~m} . \mathrm{u}$. for other products. The total production must be at least 750 units. Table 1 shows manufacturing data which are fixed.

\begin{tabular}{|l|ccccc|}
\hline & $P_{1}$ & $P_{2}$ & $P_{3}$ & $P_{4}$ & $P_{5}$ \\
\hline Raw material with capacity 1500 units & 1 & 4 & 2 & 3 & 2 \\
Machines with capacity 1600 h & 2 & 1.5 & 0.5 & 1.2 & 0.2 \\
Capital employed per unit of product & 20 & 25 & 30 & 22 & 11 \\
Sales price per unit of product (m.u.) & 120 & 130 & 90 & 110 & 70 \\
Gross profit per unit of product (m.u.) & 11 & 9 & 10 & 6 & 10 \\
Inventory cost per unit of product (m.u.) & 2 & 1 & 1.5 & 2 & 1.8 \\
\hline
\end{tabular}

Table 1: Practical example data (per unit)

Each decision-maker tends to maximize his objective function: the decision maker at the highest level maximizes the ratio of the total net profit (the difference between the gross profit and the total inventory costs and total investment in promotion) and total invested capital, while decision-makers at the lower level maximize the ratio of the total inventory of products and total production, and the ratio of the total investment in promotion and the total revenue, respectively.

Let $x_{1}, x_{2}, x_{3}, x_{4}, x_{5}$ be production quantities, $i_{1}, i_{2}, i_{3}, i_{4}, i_{5}$ stock quantities and $p_{1}, p_{2}, p_{3}, p_{4}, p_{5}$ investments in the promotion of products $P_{1}, P_{2}, P_{3}, P_{4}, P_{5}$ respectively. Based on the above data, we can form the DBL-MOLFP model:

$$
\begin{aligned}
& \underset{(\mathbf{x}, \mathbf{i}, \mathbf{p}) \in \mathbf{S}}{\max \mathbf{z}_{0}}=\frac{11 x_{1}+9 x_{2}+10 x_{3}+6 x_{4}+10 x_{5}-2 i_{1}-i_{2}-1.5 i_{3}-2 i_{4}-1.8 i_{5}-p_{1} \cdots-p_{5}}{20 x_{1}+25 x_{2}+30 x_{3}+22 x_{4}+11 x_{5}} \\
& \underset{(\mathbf{x}, \mathbf{i}, \mathbf{p}) \in \mathbf{S}}{\max \mathbf{z}_{1}}=\frac{i_{1}+i_{2}+i_{3}+i_{4}+i_{5}}{x_{1}+x_{2}+x_{3}+x_{4}+x_{5}} \\
& \underset{(\mathbf{x}, \mathbf{i}, \mathbf{p}) \in \mathbf{S}}{\max \mathbf{z}_{2}}=\frac{p_{1}+p_{2}+p_{3}+p_{4}+p_{5}}{120 x_{1}+130 x_{2}+90 x_{3}+140 x_{4}+70 x_{5}}
\end{aligned}
$$

where $\mathbf{S}$ is: 


$$
\begin{aligned}
\mathbf{S}=\{ & x_{1}+4 x_{2}+2 x_{3}+3 x_{4}+2 x_{5} \leq 1500,2 x_{1}+1.5 x_{2}+0.5 x_{3}+1.2 x_{4}+0.2 x_{5} \leq 1600 \\
& 20 x_{1}+25 x_{2}+30 x_{3}+22 x_{4}+15 x_{5} \leq 22000,0.12\left(x_{1}+x_{2}+x_{3}+x_{4}+x_{5}\right) \leq\left(i_{1}+\right. \\
& \left.i_{2}+i_{3}+i_{4}+i_{5}\right), 0.14 x_{1} \leq i_{1}, 0.11 x_{2} \leq i_{2}, 0.09 x_{3} \leq i_{3}, 0.15 x_{4} \leq i_{4}, 0.13 x_{5} \leq i_{5} \\
& 0.5\left(x_{1}+x_{2}+x_{3}+x_{4}+x_{5}\right) \geq\left(i_{1}+i_{2}+i_{3}+i_{4}+i_{5}\right), 0.44 x_{1} \geq i_{1}, 0.56 x_{2} \geq i_{2} \\
& 0.52 x_{3} \geq i_{3}, 0.48 x_{4} \geq i_{4}, 0.5 x_{5} \geq i_{5}, i_{1}, i_{2}, i_{3}, i_{4}, i_{5} \leq 100 \\
& 2.2 x_{1}+1.8 x_{2}+2 x_{3}+1.2 x_{4}+2 x_{5} \geq p_{1}+p_{2}+p_{3}+p_{4}+p_{5}, \quad 2.42 x_{1} \geq p_{1} \\
& 1.62 x_{2} \geq p_{2}, 2.4 x_{3} \geq p_{3}, 1.2 x_{4} \geq p_{4}, 1.9 x_{5} \geq p_{5}, p_{1} \leq 800 \\
& p_{1}, p_{2}, p_{3}, p_{4}, p_{5} \leq 400, x_{1}+x_{2}+x_{3}+x_{4}+x_{5} \geq 750 \\
& \left.x_{1}, x_{2}, x_{3}, x_{4}, x_{5}, i_{1}, i_{2}, i_{3}, i_{4}, i_{5}, p_{1}, p_{2}, p_{3}, p_{4}, p_{5} \geq 0\right\}
\end{aligned}
$$

\begin{tabular}{|c|c|c|c|c|}
\hline & objective function values & $\mathrm{z}_{0}$ & $\mathbf{z}_{1}$ & $\mathrm{z}_{2}$ \\
\hline $\max _{(\mathbf{x}, \mathbf{i}, \mathbf{p}) \in \mathbf{S}} \mathbf{z}_{\mathbf{0}}$ & $\begin{array}{l}x_{1}=15, x_{2}=50, x_{3}=50, x_{4}=50, x_{5}=450, \\
i_{1}=21, i_{2}=5, i_{3}=5, i_{4}=8, i_{5}=49 \\
p_{1}=p_{2}=p_{3}=p_{4}=p_{5}=0\end{array}$ & 0.6138 & 0.1293 & 0.0000 \\
\hline $\max _{(\mathbf{x}, \mathbf{i}, \mathbf{p}) \in \mathbf{z}} \mathbf{z}_{1}$ & $\begin{array}{l}x_{1}=291, x_{2}=93, x_{3}=128, x_{4}=104, x_{5}=133 \\
i_{1}=50, i_{2}=52, i_{3}=67, i_{4}=50, i_{5}=67 \\
p_{1}=p_{2}=p_{3}=p_{4}=p_{5}=0\end{array}$ & 0.4333 & 0.3818 & 0.0000 \\
\hline $\max _{(\mathbf{x}, \mathbf{i}, \mathbf{p}) \in \mathbf{S}} \mathbf{z}_{2}$ & $\begin{array}{l}x_{1}=229, x_{2}=50, x_{3}=167, x_{4}=50, x_{5}=254 \\
i_{1}=30, i_{2}=5, i_{3}=15, i_{4}=8, i_{5}=33 \\
p_{1}=555, p_{2}=81, p_{3}=400, p_{4}=60, p_{5}=400\end{array}$ & 0.3954 & 0.1240 & 0.0207 \\
\hline
\end{tabular}

Using the Charnes-Cooper model, we obtain the maximum solutions of the objective functions $\mathbf{z}_{\mathbf{0}}, \mathbf{z}_{\mathbf{1}}$ and $\mathbf{z}_{\mathbf{2}}$ on the given set $\mathbf{S}$ presented in Table 2.

Table 2: Payoff values

Table 2 indicates the conflict between objective functions $\mathbf{z}_{\mathbf{0}}, \mathbf{z}_{\mathbf{1}}$ and $\mathbf{z}_{\mathbf{2}}$. Decision-maker $D M_{0}$ makes a decision first, leading to a small degree of achievement of the objective functions of decision-makers $D M_{1}$ and $D M_{2}$. Decision-makers at the lower level $\left(D M_{1}\right.$ and $\left.D M_{2}\right)$ then maximize their objective functions, which causes a major reduction in the fulfillment of the upper level objective function. The decision-makers are consequently encouraged to cooperate, which should produce an acceptable degree of satisfaction at both levels and lead to the acceptable functioning of the entire business system [14].

According to the algorithm of the proposed methodology, decision-makers determine the aspiration levels for their objective functions, while the decision-maker at the higher level determines the positive and negative deviations from the value of the decision variables which he controls, as shown in Table 3.

\begin{tabular}{|c|ccccccccc|}
\hline & $\overline{\mathbf{z}}_{0}$ & $\overline{\mathbf{z}}_{1}$ & $\overline{\mathbf{z}}_{2}$ & $t_{01}^{L}$ & $t_{01}^{R}$ & $t_{02}^{R}$ & $t_{03}^{R}$ & $t_{04}^{R}$ & $t_{05}^{L}$ \\
\hline Aspiration level & 0.6138 & 0.3818 & 0.0207 & 50 & 150 & 150 & 150 & 150 & 100 \\
\hline
\end{tabular}

Table 3: Acceptable objective function value and acceptable positive and negative deviations of decision variables $\left(t_{02}^{L}=t_{03}^{L}=t_{04}^{L}=t_{05}^{R}=0\right)$ 
The objective function weights are calculated using AHP method as follows:

$$
\begin{array}{cccc} 
& z_{0} & z_{1} & z_{2} \\
z_{0} & 1 & 2 & 2 \\
z_{1} & 1 / 2 & 1 & 1 \\
z_{2} & 1 / 2 & 1 & 1 \\
\hline \sum_{j} e_{j} & 2 & 4 & 4
\end{array}
$$

$$
\begin{array}{cccc} 
& z_{0} & z_{1} & z_{2} \\
z_{0} & 0.50 & 0.50 & 0.50 \\
z_{1} & 0.25 & 0.25 & 0.25 \\
z_{2} & 0.25 & 0.25 & 0.25
\end{array}
$$

$$
\left[\begin{array}{l}
w_{0} \\
w_{1} \\
w_{2}
\end{array}\right]=\left[\begin{array}{l}
0.50 \\
0.25 \\
0.25
\end{array}\right]
$$

The linearized objective functions look like this:

$$
\begin{aligned}
\mathbf{z}_{\mathbf{0}, \text { lin }}= & 1.276 x_{1}-6.345 x_{2}-8.414 x_{3}-7.504 x_{4}-3.248 x_{5}-2 i_{1}-i_{2}-1.5 i_{3}-2 i_{4} \\
& -1.5 i_{5}-p_{1}-p_{2}-p_{3}-p_{4}-p_{5} \\
\mathbf{z}_{\mathbf{1}, \text { lin }}= & 0.3818\left(-x_{1}-x_{2}-x_{3}-x_{4}-x_{5}\right)+i_{1}+i_{2}+i_{3}+i_{4}+i_{5} \\
\mathbf{z}_{2, \text { lin }}= & -1.276 x_{1}-2.689 x_{2}-1.862 x_{3}-2.870 x_{4}-1.448 x_{5}+p_{1}+p_{2}+p_{3}+p_{4}+p_{5}
\end{aligned}
$$

Using forms in eq.(4) and eq.(5) and calculations $\mathbf{z}_{\mathbf{0}, \text { lin }}^{\max }=-2923.25, \mathbf{z}_{\mathbf{0}, \text { lin }}^{\min }=-4867.44$, $\mathbf{z}_{1, \text { lin }}^{\max }=-524.08, \mathbf{z}_{1, \text { lin }}^{\min }=-605.80, \mathbf{z}_{\mathbf{2}, \text { lin }}^{\max }=-246.91, \mathbf{z}_{\mathbf{2}, \text { lin }}^{\min }=-1350.98$ the membership functions of the linearized objective functions and decision variables $x_{o j}, j=1,2, \ldots, 5$ are calculated:

$$
\begin{array}{ll}
\mu_{\mathbf{z}_{\mathbf{0}, \text { lin }}}=\left(\mathbf{z}_{\mathbf{0}, \text { lin }}+4867.44\right) / 1944.19, & \text { for }-4876.44 \leq \mathbf{z}_{\mathbf{0}, \text { lin }} \leq-2923.25 \\
\mu_{\mathbf{z}_{1, \text { lin }}}=\left(\mathbf{z}_{\mathbf{1}, \text { lin }}+605.80\right) / 81.72, & \text { for }-605.80 \leq \mathbf{z}_{\mathbf{1}, \text { lin }} \leq-524.08 \\
\mu_{\mathbf{z}_{\mathbf{2}, \text { lin }}}=\left(\mathbf{z}_{\mathbf{2}, \text { lin }}+1350.98\right) / 1597.89, & \text { for }-1350.98 \leq \mathbf{z}_{\mathbf{2}, \text { lin }} \leq-246.91
\end{array}
$$

The FLGP model of the DBL-MOLFP problem looks like this:

$$
\begin{aligned}
& \underset{(\mathbf{x}, \mathbf{i}, \mathbf{p}, \mathbf{d}, \lambda) \in \mathbf{S}_{2}}{\max } \lambda \\
& \mathbf{S}_{\mathbf{2}}=\left\{(\mathbf{x}, \mathbf{i}, \mathbf{p}, \mathbf{d}, \lambda):(\mathbf{x}, \mathbf{i}, \mathbf{p}) \in \mathbf{S} \cap\left\{1.276 x_{1}-6.345 x_{2}-8.414 x_{3}-7.504 x_{4}-3.248 x_{5}\right.\right. \\
&-2 i_{1}-i_{2}-1.5 i_{3}-2 i_{4}-1.5 i_{5}-p_{1}-p_{2}-p_{3}-p_{4}-p_{5}+1944.19 d_{0}^{-}=-2923.25, \\
& 0.3818\left(-x_{1}-x_{2}-x_{3}-x_{4}-x_{5}\right)+i_{1}+i_{2}+i_{3}+i_{4}+i_{5}+81.72 d_{1}^{-}=-524.08, \\
&-1.276 x_{1}-2.689 x_{2}-1.862 x_{3}-2.870 x_{4}-1.448 x_{5}+p_{1}+p_{2}+p_{3}+p_{4}+p_{5} \\
&+ 1597.89 d_{2}^{-}=-246.91, \quad \lambda-d_{k}^{-} \leq 0, \lambda+w_{k} d_{k}^{-} \leq 1, \quad k=0,1,2 \\
& \lambda \leq\left(x_{1}-100\right) / 50, \quad \lambda \leq\left(300-x_{1}\right) / 150, \quad \lambda \leq\left(200-x_{2}\right) / 150, \\
& \lambda \leq\left(200-x_{3}\right) / 150, \quad \lambda \leq\left(200-x_{4}\right) / 150 \quad \lambda \leq\left(x_{5}-300\right) / 100, \\
&\left.\left.x_{1}, x_{2}, x_{3}, x_{4}, x_{5}, i_{1}, i_{2}, i_{3}, i_{4}, i_{5}, p_{1}, p_{2}, p_{3}, p_{4}, p_{5}, d_{0}^{-}, d_{1}^{-}, d_{2}^{-}, \lambda \geq 0\right\}\right\}
\end{aligned}
$$

The following solutions are obtained: $\left(x_{1}, x_{2}, x_{3}, x_{4}, x_{5}\right)=(150,50,116,50,384),\left(i_{1}, i_{2}, i_{3}\right.$, $\left.i_{4}, i_{5}\right)=(38,7,60,24,50),\left(p_{1}, p_{2}, p_{3}, p_{4}, p_{5}\right)=(363,81,157,0,0), \lambda=0.55528,\left(\mathbf{z}_{0}, \mathbf{z}_{1}, \mathbf{z}_{2}\right)=$ $(0.497,0.239,0.008),\left(\mu_{0}, \mu_{1}, \mu_{2}\right)=(0.47,0.45,0.42)$.

Model in eq.(9) is for comparison, subsequently solved by using goal programming methodology [10]. The same data are used as input as in the previous methodology. The following solutions are obtained: $\left(x_{1}, x_{2}, x_{3}, x_{4}, x_{5}\right)=(357,50,50,50,243),\left(i_{1}, i_{2}, i_{3}, i_{4}, i_{5}\right)=$ $(50,6,5,8,32),\left(p_{1}, p_{2}, p_{3}, p_{4}, p_{5}\right)=(0,0,120,60,208),\left(\mathbf{z}_{0}, \mathbf{z}_{1}, \mathbf{z}_{2}\right)=(0.51467,0.13467,0.00498)$, $\left(\mu_{0}, \mu_{1}, \mu_{2}\right)=(0.55,0.04,0.24)$.

Comparing the obtained solutions, it can be concluded that they differ significantly, even though the same parameters are used in both methodologies. However, the FGP methodology gives more uniform membership function values of the objective functions than the goal programming methodology. 


\section{Conclusion}

Choosing a methodology for solving DBL-MOLFP problems is very demanding. In this paper, we propose a FGP methodology to solve DBL-MOLFP problems. The proposed methodology was tested using an example of an fictitious company's the production plan, inventory products and promotion cost determination. The results obtained indicate the possibility of using the proposed methodology to solve such problems. The proposed methodology can be simply applied to solve real problems. The results obtained also indicate the advantages of the proposed methodology related to the GP methodology.

For future research, we recommend testing the efficiency of the proposed methodology to solve more practical DBL-MOLFP examples and its further development.

\section{References}

[1] Ahlatcioglu, M. and Tiryaki, F. (2007). Interactive Fuzzy Programming for Decentralized Two-Level Linear Fractional Programming (DTLLFP) Problems. Omega, 35(4), 432-450. doi: 10.1016/j.omega.2005.08.005

[2] Anandalingam, G. (1988). A Mathematical Programming Model of the Decentralized MultiLevel Systems. The Journal of Operational Research Society, 39(11), 1021-1033. doi: 10.1057 /jors. 1988.172

[3] Baky, I. A. (2009). Fuzzy goal programming algorithm for solving decentralized bi-level multi-objective programming problems. Fuzzy Sets and Systems, 160(18), 2701-2713. doi: 10.1016/j.fss.2009.02.022

[4] Baky, I. A. (2010). Solving multi-level multi-objective linear programming problems through fuzzy goal programming approach. qApplied Mathematical Modelling, 34(9), 2377-2387. doi: 10.1016/j.apm.2009.11.004

[5] Emam, O. E. (2013). Interactive approach to bi-level integer multi-objective fractional programming problem. Applied Mathematics and Computation, 223, 17-24. doi: 10.1016/j.amc.2013.07.085

[6] Hansen, P., Jaumard, B. and Savard, G. (1992). New Branch-and-Bound Rules for Linear Bilevel Programming. SIAM Journal on Scientific and Statistical Computing, 13(5), 1194-1217. doi: $10.1137 / 0913069$

[7] Lachhwani, K. (2015). Modified FGP approach for multilevel multi objective linear fractional programming problems, Applied Mathematics and Computation, 266, 1038-1049. doi: 10.1016/j.amc.2015.06.027

[8] Lachhwani, K. and Poonia, M. P. (2012). Mathematical solution of multi level fractional programming problem with fuzzy goal programming approach. , 8(16), 1-11. doi: 10.1186/2251-712x-8-16

[9] Mishra, S. (2007). Weighting method for bi-level linear fractional programming problems. European Journal of Operational Research, 183(1), 296-302. doi: 10.1016/j.ejor.2006.09.053

[10] Mohamed R. H. (1997). The relationship between goal programming and fuzzy programming. Fuzzy Sets and Systems, 89(2), 215-222. doi: 10.1016/s0165-0114(96)00100-5

[11] Osman, M. S., Emam, O. E. and Elsayed, M. A. (2018). Interactive Approach for Multi-Level Multi-Objective Fractional Programming Problems with Fuzzy Parameters. Beni-Suef University Journal of Basic and Applied Sciences, 7(1), 139-149. doi: 10.1016/j.bjbas.2017.08.005

[12] Perić, T. and Babić, Z. (2014). A new goal programming approach for multiobjective fractional linear programming. Proceedings of the 25th DAAAM International Symposium on Inteligent Manufacturing and Automation, 25(1), 1-10.

[13] Perić, T., Babić, Z. and Omerović, M. (2017). Analysis of the efficiency of the linearization techniques for solving multi-objective linear fractional programming problems by goal programming. Croatian Operational Research Review, 8(1), 249-264. doi: 10.17535/crorr.2017.0016

[14] Perić, T. and Babić, Z. (2012). Financial structure optimization by using a goal programming approach. Croatian Operational Research Review, 3(1), 150-162. https://hrcak.srce.hr/96811

[15] Pramanik, S. and Roy, T. K. (2007). Fuzzy goal programming approach to multilevel programming problems. European Journal of Operational Research, 176(2), 1151-1166. doi: 10.1016/j.ejor.2005.08.024 
[16] Sakawa, M. and Nishizaki, I. (2001). Interactive fuzzy programming for two-level linear fractional programming problems. Fuzzy Sets and Systems, 119(1), 31-40. doi: 10.1016/s01650114(99)00066-4

[17] Sakawa, M., Nishizaki, I. (2002). Interactive fuzzy programming for decentralized two-level linear programming problems. Fuzzy Sets and Systems, 125(3), 301-315. doi: 10.1016/s01650114(01)00042-2

[18] Shih, H. S., Lai, Y. J. and Lee, E. S. (1996). Fuzzy approach for multi-level programming problems. Computers and Operations Research, 23(1), 73-91. doi: 10.1016/0305-0548(95)00007-9

[19] Shih, H. S. and Lee, E. S. (2000). Compensatory Fuzzy Multiple Decision Making. Fuzzy Sets and Systems, 114(1), 71-87. doi: 10.1016/s0165-0114(98)00409-6

[20] Sinha, A., Malo, P. and Deb, K. (2018). A Review on Bilevel Optimization: From Classical to Evolutionary Approaches and Applications. IEEE Transactions on Evolutionary Computation, 22(2), 276-295. doi: 10.1109/tevc.2017.2712906

[21] Sinha, S. (2003). Fuzzy programming approach to multi-level programming problems. Fuzzy Sets and Systems, 136(2), 189-202. doi: 10.1016/s0165-0114(02)00362-7

[22] Sinha, S., Sinha S. B. (2002). KKT transformation approach for multi-objective multi-level linear programming problems. European Journal of Operational Research, 143(1), 19-31. doi: 10.1016/s0377-2217(01)00323-x

[23] Stackelberg, H. (1952). The theory of the market economy. New York: Oxford University Press.

[24] Toksari, M. D. and Bilim, Y. (2015). Interactive Fuzzy Goal Programming Based on Jacobian Matrix to Solve Decentralized Bi-level Multi-objective Fractional Programming Problems. International Journal of Fuzzy Systems, 17(4), 499-508. doi: 10.1007/s40815-015-0036-1 